\title{
Accomodating Irregular Subdomains in Domain Decomposition Theory
}

\author{
Olof B. Widlund ${ }^{1}$ \\ Courant Institute, 251 Mercer Street, New York, NY 10012, USA \\ widlund@cims.nyu.edu
}

Summary. In the theory for domain decomposition methods, it has previously often been assumed that each subdomain is the union of a small set of coarse shaperegular triangles or tetrahedra. Recent progress is reported, which makes it possible to analyze cases with irregular subdomains such as those produced by mesh partitioners. The goal is to extend the analytic tools so that they work for problems on subdomains that might not even be Lipschitz and to characterize the rates of convergence of domain decomposition methods in terms of a few, easy to understand, geometric parameters of the subregions. For two dimensions, some best possible results have already been obtained for scalar elliptic and compressible and almost incompressible linear elasticity problems; the subdomains should be John or Jones domains and the rates of convergence are determined by parameters that characterize such domains and that of an isoperimetric inequality. Technical issues for three dimensional problems will also be discussed.

\section{Introduction}

In developing theory for domain decomposition methods of iterative substructuring type, we have typically assumed that each subdomain is quite regular, e.g., the union of a small set of coarse triangles or tetrahedra; see, e.g., [Toselli and Widlund, 2005, Assumption 4.3]; we will call such subdomains regular. However, such an assumption is unlikely to hold especially if the subdomains result from using a mesh partitioner, such as METIS, see Karypis and Kumar [1998]. Then, the subdomain boundaries might not even be uniformly Lipschitz continuous in the sense that the number of patches required to cover $\partial \Omega$, and in each of which the boundary is the graph of a Lipschitz continuous function, is not uniformly bounded independently of the finite element mesh size. We also note that the shape of the subdomains are likely to change if the mesh size is altered and a mesh partitioner is used repeatedly.

The purpose of this paper is to report on recent development of theory for domain decomposition methods under very weak assumptions on the subdomain partitioning and to categorize the rates of convergence of the algorithms in terms of a few geometric parameters. This work is being carried out in collaboration with Clark R. Dohrmann, Axel Klawonn, and Oliver Rheinbach and has so far resulted in three 
archival papers, Dohrmann et al. [2008], Klawonn et al. [2007], and Dohrmann and Widlund [2008]. Results have been obtained for scalar elliptic problems, compressible linear elasticity, and almost incompressible elasticity problems approximated by mixed finite elements with pressure spaces with discontinuous elements.

We will denote a set of nonoverlapping subdomains by $\left\{\Omega_{i}\right\}$. Their closures cover the given domain $\Omega$, and the interface between them is denoted by $\Gamma$. We will discuss results for the FETI-DP and BDDC families of algorithms, defined on such a set of nonoverlapping subdomains, as well as results for some two-level Schwarz algorithms based on overlapping subdomains $\Omega_{i}^{\prime}$. We will assume that each such subdomain has been obtained from one of the $\Omega_{i}$ by adding one or more layers of finite elements. The FETI-DP and BDDC algorithms are iterative substructuring algorithms, i.e., they provide preconditioners based on nonoverlapping subdomains.

So far, complete results have only been obtained for problems in the plane. We will consider scalar elliptic problems of the following form:

$$
-\operatorname{div}(\rho(x) \nabla u(x))=f(x) \quad x \in \Omega,
$$

with a homogeneous Dirichlet boundary condition on $\partial \Omega$; we make this choice of boundary condition just to simplify the discussion of our results. The coefficient $\rho(x)$ is strictly positive and assumed to be equal to a constant $\rho_{i}$ for $x \in \Omega_{i}$, but is otherwise arbitrary. As is often the case, our results hold equally well for isotropic compressible elasticity problems.

$$
-\operatorname{div}(2 \mu \varepsilon(\mathbf{u})+\lambda \operatorname{tr}(\varepsilon(\mathbf{u})) I)=f \quad \text { in } \Omega \subset \mathbb{R}^{n} .
$$

Here $\varepsilon_{i j}(\mathbf{u})=(1 / 2)\left(\partial u_{i} / \partial x_{j}+\partial u_{j} / \partial x_{i}\right)$ and $\mu$ and $\lambda$ the Lamé parameters; in the almost incompressible case, $\lambda / \mu$ takes on very large values.

Older results on domain decomposition methods for linear elasticity are summarized in [Toselli and Widlund, 2005, Chapter 8] and Klawonn and Widlund [2006] gives more recent results on FETI-DP algorithms. All this work is for regular subdomains. We note that there are extensive and successful numerical results for more general problems; see, e.g., Klawonn and Rheinbach [2006], Klawonn and Rheinbach [2007], and Dohrmann et al. [2007b].

We use lower order, continuous finite elements and triangulations with shape regular elements, i.e., the diameter of an element is bounded uniformly by a constant times the radius of the largest inscribed circle or sphere and assume that each subdomain is a union of elements. For almost incompressible elasticity, we use an inf-sup stable pair of finite element spaces after introducing the new variable $p=-\lambda \operatorname{div} \mathbf{u}$ and assume that the elements of this second finite element space are discontinuous. We can then eliminate this pressure variable element-wise, recover a positive definite problem, and use the same conjugate gradient acceleration as for compressible elasticity.

For a collection of auxiliary results used in the analysis of iterative substructuring algorithms, in the case of regular subdomains, see [Toselli and Widlund, 2005, Section 4.6]. Our studies require the generalization of these technical tools to obtain proofs of bounds on the convergence rates of FETI-DP algorithms and on certain overlapping Schwarz methods for less regular subdomains. We also have had to modify some of the reasoning in the main proofs. Four auxiliary results, namely a Poincaré inequality, a Sobolev-type inequality for finite element functions, bounds for certain edge and face terms, and a finite element extension theorem are required 
in our proofs; see Lemmas 2, 6, 7, and 8. We will work with John domains and Jones domains, see Definitions 1 and 2; the latter are also known as uniform or $(\varepsilon, \infty)$ domains. We will express our bounds on the rate of convergence of our algorithm in terms of the few parameters of Definitions 1, 2, and Lemma 1.

\section{A Poincaré inequality, John and Jones domains}

We will first introduce John domains and then consider a Poincaré inequality for such domains. We will also introduce Jones domains; the latter are needed in order to obtain a finite element extension theorem, required in the analysis of FETI and BDDC algorithms, see Klawonn et al. [2007], but not for the algorithms based on overlapping subdomains, see Dohrmann et al. [2007a,b, 2008], and Dohrmann and Widlund [2008].

We next give a definition of a John domain; see Hajłasz [2001] and the references therein. In the proofs of several of our auxiliary results, we will assume that the subdomains belong to this class.

Definition 1 (John domain). A domain $\Omega \subset \mathbb{R}^{n}-$ an open, bounded, and connected set - is a John domain if there exists a constant $C_{J} \geq 1$ and a distinguished central point $x_{0} \in \Omega$ such that each $x \in \Omega$ can be joined to it by a rectifiable curve $\gamma:[0,1] \rightarrow \Omega$ with $\gamma(0)=x_{0}, \gamma(1)=x$ and $|x-\gamma(t)| \leq C_{J} \cdot \operatorname{distance}(\gamma(t), \partial \Omega)$ for all $t \in[0,1]$.

This condition can be viewed as a twisted cone condition. We note that certain snowflake curves with fractal boundaries are John domains and that the length of the boundary of a John domain can be arbitrarily much longer than its diameter. We also note that for any choice of the point $x_{0}$, there is a point $x \in \Omega$ at a distance of at least diameter $(\Omega) / 2$. We find that diameter $(\Omega) \leq 2 C_{J} r_{\Omega}$, where $r_{\Omega}$ is the radius of the largest ball inscribed in $\Omega$ and centered at $x_{0}$. Conditions on the boundary are of course also imposed.

In any analysis of any domain decomposition method with a second, coarse level, we need a Poincaré inequality. This inequality is closely related to an isoperimetric inequality. The next lemma is attributed to Maz'ja [1960] and Federer and Fleming [1960].

Lemma 1 (Isoperimetric inequality). Let $\Omega \subset \mathbb{R}^{n}$ be a domain and let $u$ be sufficiently smooth. Then,

$$
\inf _{c \in R}\left(\int_{\Omega}|u-c|^{n /(n-1)} d x\right)^{(n-1) / n} \leq \gamma(\Omega, n) \int_{\Omega}|\nabla u| d x
$$

if and only if,

$$
[\min (|A|,|B|)]^{1-1 / n} \leq \gamma(\Omega, n)|\partial A \cap \partial B| .
$$

Here, $A \subset \Omega$ is an arbitrary open set, and $B=\Omega \backslash \bar{A} ; \gamma(\Omega, n)$ is the best possible constant and $|A|$ is the measure of the set $A$, etc.

We note that the domain does not need to be star-shaped or Lipschitz. For $n=2$, the best choice of $c=\bar{u}_{\Omega}$, the average of $u$ over the domain. A small value of $\gamma(\Omega, n)$ is desirable for our purposes. 
It is known that any simply connected plane domain with a finite Poincaré parameter $\gamma(\Omega, 2)$ is a John domain; see Buckley and Koskela [1995]. It is also known, see Bojarski [1988], that any John domain has a bounded Poincaré parameter $\gamma(\Omega, n)$.

We obtain the standard Poincaré inequality by using the Cauchy-Schwarz inequality, for two dimensions, and the Hölder inequality several times for three.

Lemma 2 (Poincaré's inequality). Let $\Omega$ be a John domain. Then,

$$
\left\|u-\bar{u}_{\Omega}\right\|_{L^{2}(\Omega)}^{2} \leq(\gamma(\Omega, n))^{2}|\Omega|^{2 / n}\|\nabla u\|_{L^{2}(\Omega)}^{2} \quad \forall u \in H^{1}(\Omega) .
$$

Throughout, we will use a weighted $H^{1}\left(\Omega_{i}\right)$-norm defined by

$\|u\|_{H^{1}\left(\Omega_{i}\right)}^{2}:=\int_{\Omega_{i}} \nabla u \cdot \nabla u d x+1 / H_{i}^{2} \int_{\Omega_{i}}|u|^{2} d x=|u|_{H^{1}\left(\Omega_{i}\right)}^{2}+1 / H_{i}^{2} \int_{\Omega_{i}}|u|^{2} d x$.

Here $H_{i}$ is the diameter of $\Omega_{i}$. The weight for the $L^{2}$-term results from the standard $H^{1}$-norm on a domain with diameter one and a dilation. We use Lemma 2 to remove $L^{2}$-terms in some of our estimates.

We next consider Jones domains.

Definition 2 (Jones domains). A domain $\Omega \subset \mathbb{R}^{n}$ is a Jones domain if there exists a constant $C_{U}$ such that any pair of points $x_{1} \in \Omega$ and $x_{2} \in \Omega$ can be joined by a rectifiable curve $\gamma(t):[0,1] \rightarrow \Omega$ with $\gamma(0)=x_{1}, \gamma(1)=x_{2}$, and where the Euclidean arc length of $\gamma \leq C_{U}\left|x_{1}-x_{2}\right|$ and $\min _{i=1,2}\left|x_{i}-\gamma(t)\right| \leq C_{U}$. distance $(\gamma(t), \partial \Omega)$ for all $t \in[0,1]$.

It is known, and easy to see, that any Jones domain is a John domain. It is also easy to construct John domains that are not Jones domains. According to [Jones, 1981, Theorem 4], they form the largest class of finitely connected domains for which an extension theorem holds in two dimensions. It is also known that every Jones domain $\mathbb{R}^{n}$ allows for a bounded extension with respect to the seminorm of $H^{1}$, see [Jones, 1981, Theorem 2].

Lemma 3. Let $\Omega \subset \mathbb{R}^{n}$ be a Jones domain and let $P_{0}$ be the space of constants. There then exists a bounded, linear operator $E_{\Omega}: H^{1}(\Omega) / P_{0} \rightarrow H^{1}\left(\mathbb{R}^{n}\right) / P_{0}$, which extends any element in $H^{1}(\Omega)$ to one defined for all of $\mathbb{R}^{n}$, i.e., $\left(E_{\Omega} u\right)_{\mid \Omega}=u \forall u \in$ $H^{1}(\Omega) / P_{0}$. The norm of this operator depends only on $C_{U}(\Omega)$.

An important tool in any study of elasticity is the second Korn inequality. For a proof for Jones domains, see Durán and Muschietti [2004].

Lemma 4 (Korn inequality for Jones domains). Let $\Omega \subset \mathbb{R}^{n}$ be a bounded Jones domain. Then, there exists a constant $C$, which depends only on the Jones constant $C_{U}(\Omega)$ and the dimension $n$, such that

$$
|\mathbf{u}|_{H^{1}(\Omega)}^{2} \leq C \sum_{i j}\left\|\varepsilon(\mathbf{u})_{i j}\right\|_{L^{2}(\Omega)}^{2}
$$

for all $\mathbf{u} \in\left\{\mathbf{u} \in \mathbf{H}^{\mathbf{1}}(\Omega): \int_{\Omega}\left(\frac{\partial u_{i}}{\partial x_{j}}-\frac{\partial u_{j}}{\partial x_{i}}\right) d x=0, i, j=1, \ldots, n\right\}$. 
Their proof has many details in common with Jones' proof of Lemma 3. In the case of mixed finite element approximations of almost incompressible elasticity, we also need to establish the inf-sup stability of the mixed method. This problem is closely related to the Korn inequality; see, e.g., Bramble [2003] in which new proofs of both results are given for general Lipschitz domains and the continuous case. There is a proof of the underlying inequality for John domains in Acosta et al. [2006]; the constant in that estimate depends only on the John parameter $C_{J}(\Omega)$.

\section{FETI-DP and BDDC algorithms}

We first note that these two families of domain decomposition algorithms are closely related. Any such algorithm is characterized by a set of primal constraints and it is known that a pair of FETI-DP and BDDC algorithms, with the same primal constraints, have spectra which are almost identical; these spectra determine the rate of convergence of these preconditioned conjugate gradient methods. This result was first established in Mandel et al. [2005]; see also Li and Widlund [2006] for a simpler proof and a general discussion and general references on these algorithms.

We denote by $W^{h}\left(\Omega_{i}\right)$ the standard finite element space of continuous, piecewise linear functions on $\Omega_{i}$ which vanish on $\partial \Omega_{i} \cap \partial \Omega$. We will denote by $h_{i}$ the smallest diameter of the finite elements in the subdomain $\Omega_{i}$. The corresponding finite element trace spaces are denoted by $W^{(i)}:=W^{h}\left(\partial \Omega_{i} \cap \Gamma\right), i=1, \ldots, N$. The product space of the $W^{(i)}$ is denoted by $W$ and in the context of these iterative methods, we need to consider elements of this space, which are not necessarily continuous across the interface. However, the primal variables are global. Thus, in two dimensions, the values at the subdomain vertices are often chosen to be primal, i.e., to have common values. In the FETI-DP algorithms, the remaining continuity requirements at all the remaining nodes on the interface will only be fully in force when the iteration has converged and certain Lagrange multipliers have reached their correct values. In the BDDC algorithms, on the other hand, continuity across the interface is restored, in each step of the iteration, by replacing the discontinuous values on the interface by a weighted average. The partially assembled subspace, with the primal variables global, will be denoted by $\widetilde{W}$.

We can now formulate our main result, which is also valid for compressible elasticity.

Theorem 1 (Condition number estimate). Let the domain $\Omega \subset \mathbb{R}^{2}$ be partitioned into subdomains $\Omega_{i}$, which are partitioned into shape regular elements and which have complements $\mathcal{C} \Omega_{i}$ that are Jones domains. Let all values at the subdomain vertices be primal. Then, with $M$ the Dirichlet preconditioner, $F$ the FETI-DP operator, the condition number of the preconditioned conjugate gradient method satisfies

$$
\kappa\left(M^{-1} F\right) \leq C \max _{i}\left(1+\log \left(H_{i} / h_{i}\right)\right)^{2} .
$$

Here $C$ is a constant which depends only on the parameters $C_{J}\left(\Omega_{i}\right)$ and $C_{U}\left(\mathcal{C} \Omega_{i}\right)$ of Definitions 1 and 2, the Poincaré parameters $\gamma\left(\Omega_{i}, 2\right)$ of the subdomains, and the shape regularity of the finite elements. The result is also independent of possible jumps in the coefficient $\rho_{i}$, or the Lamé parameters, across the interface between the subdomains. 
A complete proof of this result is given in Klawonn et al. [2007]. It is as strong a result as those in Klawonn et al. [2002] and Klawonn and Widlund [2006] for regular subdomains. We also note that numerical experiments on very irregular snowflake subdomains have added interesting insight on how best to scale the FETIDP preconditioners.

We will now indicate what is required to establish the theorem. We denote by $\mathcal{H}$ the discrete harmonic extension operator: $\mathcal{H}\left(v_{\Gamma}\right)$ is the minimal energy extension of the restriction of the finite element function $v$ to the interface $\Gamma$. In what follows, $\mathcal{H}(u v)$ will mean the discrete harmonic extension of the finite element function obtained by interpolating the product of $u$ and $v$. For each edge $\mathcal{E}^{i j}$ - the open set common to $\partial \Omega_{i}$ and $\partial \Omega_{j}$ and which does not contain its endpoints - we define an edge cutoff function $\theta_{\mathcal{E}^{i j}}$, which is the discrete harmonic function which equals 1 at all nodes on the edge $\mathcal{E}^{i j}$ and which vanishes at all other interface nodes; cf. [Toselli and Widlund, 2005, Section 4.6].

We note that part of the proof of any result on a FETI-DP or BDDC algorithm, such as Theorem 1, is purely algebraic. It is also known that in order to fully prove a result such as Theorem ??, we need to use tools of analysis to establish a result such as Lemma 5; see, e.g., Li and Widlund [2006] or [Klawonn and Widlund, 2006, Section 8]. For the set of primal constraints considered in Theorem 1, we need to prove:

Lemma 5. Let $\mathcal{E}^{i j}$ be an edge common to the boundaries of $\Omega_{i}$ and $\Omega_{j}$. For all $v \in \widetilde{W}$ and with $v^{(i)}:=R^{(i)} v, v^{(j)}:=R^{(j)} v$, we have

$$
\begin{aligned}
\rho_{i}\left|\mathcal{H}\left(\theta_{\mathcal{E}^{i j}} \delta_{i}^{\dagger}\left(v^{(i)}-v^{(j)}\right)\right)\right|_{H^{1}\left(\Omega_{i}\right)}^{2} & \leq C\left(1+\log \left(H_{i} / h_{i}\right)\right)^{2} \rho_{i}\left|v^{(i)}\right|_{H^{1}\left(\Omega_{i}\right)}^{2} \\
& +C\left(1+\log \left(H_{j} / h_{j}\right)\right)^{2} \rho_{j}\left|v^{(j)}\right|_{H^{1}\left(\Omega_{j}\right)}^{2} .
\end{aligned}
$$

Here $R^{(i)}$ denotes the restriction operator from $\widetilde{W}$ to $W^{(i)}$. The parameter $\delta_{i}^{\dagger}:=$ $\rho_{i}^{\gamma} / \sum_{j \in \mathcal{N}_{x}} \rho_{j}^{\gamma}$, where $\gamma \in[1 / 2, \infty)$ and $\mathcal{N}_{x}$ is the set of indices $j$ of the subregions with $x$ on their boundaries. The constant $C$ in the inequality depends only on the parameters $C_{J}\left(\Omega_{i}\right)$ and $C_{U}\left(\mathcal{C} \Omega_{i}\right)$ of Definitions 1 and 2, the Poincaré parameters $\gamma\left(\Omega_{i}, 2\right)$ of the subdomains, and the shape regularity of the finite elements.

To prove this lemma, we need three auxiliary results, in addition to Poincaré's inequality. The first is a discrete Sobolev inequality. This inequality, (5), is well known in the theory of iterative substructuring methods. Proofs for domains satisfying an interior cone condition are given in Bramble et al. [1986] and [Brenner and Scott, 2002, Section 4.9] and a different proof is given in [Toselli and Widlund, 2005, page 102]. For a proof for John domains, see Dohrmann et al. [2008].

Lemma 6 (Discrete Sobolev inequality). Let $\Omega_{i} \subset \mathbb{R}^{2}$ be a John domain. Then,

$$
\left\|u-\bar{u}_{\Omega_{i}}\right\|_{L^{\infty}\left(\Omega_{i}\right)}^{2} \leq C\left(1+\log \left(H_{i} / h_{i}\right)\right)|u|_{H^{1}\left(\Omega_{i}\right)}^{2},
$$

for all $u \in W^{h}\left(\Omega_{i}\right)$. The constant $C$ depends only on the John parameter $C_{J}\left(\Omega_{i}\right)$ of $\Omega_{i}$ and the shape regularity of the finite elements.

A three-dimensional counterpart of Lemma 6 is given in [Toselli and Widlund, 2005, Subsection 4.6.2]. This provides an estimate of the $L^{2}$-norm of finite element functions over subdomain edges and this result has not yet been extended fully to the case of irregular subdomains. 
Another important result provides estimates for different types of edge functions. For regular subdomains in two dimensions, this lemma was first given in Dryja and Widlund [1989].

Lemma 7 (Edge lemma). Let $\Omega_{i} \subset \mathbb{R}^{2}$ be a John domain, let $\mathcal{E}^{i j} \subset \partial \Omega_{i}$ be an edge, and $\theta_{\mathcal{E}^{i j}} \in W^{h}\left(\Omega_{i}\right)$ be the finite element function which equals 1 at all nodes of $\mathcal{E}^{i j}$, and which vanishes at all the other nodes on $\partial \Omega_{i}$, and is discrete harmonic in $\Omega_{i}$. Then, for any $u \in W^{h}\left(\Omega_{i}\right)$, we have

$$
\begin{gathered}
\left|\mathcal{H}\left(\theta_{\mathcal{E}^{i j}} u\right)\right|_{H^{1}\left(\Omega_{i}\right)}^{2} \leq C\left(1+\log \left(H_{i} / h_{i}\right)\right)^{2}\|u\|_{H^{1}\left(\Omega_{i}\right)}^{2}, \\
\left|\theta_{\mathcal{E}^{i j}}\right|_{H^{1}\left(\Omega_{i}\right)}^{2} \leq C\left(1+\log \left(H_{i} / h_{i}\right)\right)
\end{gathered}
$$

and

$$
\left\|\theta_{\mathcal{E}^{i j}}\right\|_{L^{2}\left(\Omega_{i}\right)}^{2} \leq C H_{i}^{2}\left(1+\log \left(H_{i} / h_{i}\right)\right) .
$$

Here, $C$ depends only on the John parameter $C_{J}\left(\Omega_{i}\right)$ of $\Omega_{i}$ and the shape regularity of the finite elements. The logarithmic factor in (8) can be removed if all angles of the triangulation are acute and $W^{h}$ is a space of piece-wise linear finite elements.

For a proof, see Klawonn et al. [2007].

In order to advance the work on three dimensional problems, it would be central to develop similar face and edge lemmas under some suitable geometric assumptions; cf. [Toselli and Widlund, 2005, Section 4.6] for results in case the subdomains are regular. At this time, we can prove such bounds for a subdomain which contains a Lipschitz domain with edges which are common to those of the subdomain. It is also clear that in the general case, we need a limit on the number of points on each edge since it can easily be seen that the energy of the edge function $\theta_{\mathcal{E}^{i k}}$ will grow in proportion to this number. In a case of many edge nodes, the energy of the face functions must also be large.

We establish inequality (6) by using ideas similar to those of [Toselli and Widlund, 2005, Proofs of Lemmas 4.24 and 4.25]. We construct a function $\vartheta_{\mathcal{E}^{i j}}$ which has the same boundary values as $\theta_{\mathcal{E}^{i j}}$ and which satisfies the two inequalities (6) and (7). Since $\theta_{\mathcal{E}^{i j}}$ and $\mathcal{H}\left(\theta_{\mathcal{E}^{i j}} u\right)$ are discrete harmonic, the two inequalities (6) and (7) will then hold. We note that in our work on almost incompressible elasticity, described in Section 5, such results are also required for domains with large aspect ratios; see [Dohrmann and Widlund, 2008, Lemma 5.4]. The bounds as in Lemma 7 will grow linearly with the aspect ratio of the subdomains.

The next lemma was proven for Lipschitz domains and quite general conforming finite elements in Widlund [1987], using a technique from Astrakhatsev [1978]; see also Toselli and Widlund [2005] for a different proof. In Klawonn et al. [2007], we have developed a new proof for more general domains, which uses Lemma 3 and a result by Scott and Zhang [1990]. We note that this result can be viewed as providing an estimate of the rate of convergence of the classical Dirichlet-Neumann algorithm for two subdomains and with a quite irregular interface; see, e.g., [Toselli and Widlund, 2005, Section 1.3.3].

Lemma 8 (Extension lemma). Let $\Omega_{i}$ and $\Omega_{j}$, subsets of $\mathbb{R}^{n}$, be two subdomains with a common $(n-1)$-dimensional interface $\Gamma^{i j}$. Furthermore, let $\Omega_{i}$ be a domain with a complement which is a Jones domain, let

$$
W_{i}^{h}=\left\{v_{h} \in W^{h}\left(\Omega_{i}\right): v_{h}(x)=0 \text { at all nodes of } \partial \Omega_{i} \backslash \Gamma^{i j}\right\}
$$


and let

$$
W_{j}^{h}=\left\{v_{h} \in W^{h}\left(\Omega_{j}\right): v_{h}(x)=0 \text { at all nodes of } \partial \Omega_{j} \backslash \Gamma^{i j}\right\} .
$$

Then, there exists an extension operator

$$
E_{j i}^{h}: W_{j}^{h} \longrightarrow W_{i}^{h}
$$

with the following properties:

1. $\left(E_{j i}^{h} u_{h}\right)_{\mid \Omega_{j}}=u_{h} \quad \forall u_{h} \in W_{j}^{h}$,

$$
\text { 2. }\left|E_{j i}^{h} u_{h}\right|_{H^{1}\left(\Omega_{i}\right)} \leq C\left|u_{h}\right|_{H^{1}\left(\Omega_{j}\right)} \quad \forall u_{h} \in W_{j}^{h} \text {, }
$$

where the constant $C$ depends only on the Jones parameter $C_{U}\left(\mathcal{C} \Omega_{i}\right)$ of the complement of $\Omega_{i}$ and the shape regularity of the elements and is otherwise independent of the finite element mesh sizes $h_{i}$ and $h_{j}$ and the diameters $H_{i}$ and $H_{j}$.

\section{An overlapping Schwarz method}

When we now turn to another major family of domain decomposition methods, we note that the overlapping Schwarz methods can be used even if the stiffness matrix of the problem is only available in fully assembled form. This is important in many applications. The FETI-DP and BDDC algorithms, in contrast, require access to the stiffness matrices of the subdomains.

In the case of a scalar elliptic problem with constant coefficients in each substructure, a coarse space for a problem in three dimensions can be defined as the range of the interpolation operator

$$
I_{B}^{h} u(x)=\sum_{i, \ell} u\left(\mathcal{V}^{i \ell}\right) \theta_{\mathcal{V}^{i \ell}}(x)+\sum_{i, k} \bar{u}_{\mathcal{E}^{i k}} \theta_{\mathcal{E}^{i k}}(x)+\sum_{i, j} \bar{u}_{\mathcal{F}^{i j}} \theta_{\mathcal{F}^{i j}}(x) .
$$

Here, $\bar{u}_{\mathcal{E}^{i k}}$ and $\bar{u}_{\mathcal{F}^{i j}}$ are averages over edges and faces, respectively. We obtain an analogous expression for two dimensions by dropping the face terms.

With suitable local spaces, it is known that the resulting iterative substructuring algorithm [Toselli and Widlund, 2005, Algorithm 5.16] is quite satisfactory with a condition number bound of the form $C \max _{i}\left(1+\log \left(H_{i} / h_{i}\right)\right)$. The constant $C$ is independent of the number of subdomains as well as jumps in the coefficients between the subdomains. By enriching the coarse space by basis functions constructed from the same cutoff functions and the rigid body modes similar results are possible for linear elasticity.

These coarse spaces have also recently been combined with local components based on overlapping subdomains, just as for traditional overlapping Schwarz methods; see Dohrmann et al. [2007a,b], Dohrmann and Widlund [2008]. These methods are all additive Schwarz methods and they are therefore definined in terms of a coarse subspace and many local spaces defined by subspaces of finite element functions supported in the overlapping subdomains $\Omega_{i}^{\prime}$. We note that there would be no additional technical issues should we choose to work with multiplicative or hybrid Schwarz methods as in [Toselli and Widlund, 2005, Chapter 2]. The resulting 
algorithms have already proven quite successful for very large problems and an implementation, by Clark Dohrmann, for massively parallel computing systems is now part of the Salinas software system for structural dynamics problems developed at Sandia National Laboratories, Albuquerque, NM.

In the case of Lipschitz subdomains, the weights for the face terms in an interpolation formula such as (9) can easily be bounded by using Cauchy-Schwarz's inequality and an elementary trace theorem such as [Nečas, 1967, Theorem 1.2]. For more general subdomains, this argument breaks down but the average can be replaced by any bounded functional, which depends only on the trace of the finite element function on the face, and which reproduces constants. In two dimensions, the average over an edge can simply be replaced by the maximum of the finite element function and we can then use Lemmas 6 and 7 at the expense of an additional logarithmic factor. The same approach would result in a factor $H_{i} / h_{i}$ in three dimensions. Instead the face average over $\mathcal{F}^{i j}$ can be replaced by

$$
\left(\nabla \theta_{\mathcal{F}^{i j}}, \nabla \mathcal{H}\left(\theta_{\mathcal{F}^{i j}} u\right)\right)_{L^{2}\left(\Omega_{i}\right)} /\left(\nabla \theta_{\mathcal{F}^{i j}}, \nabla \theta_{\mathcal{F}^{i j}}\right)_{L^{2}\left(\Omega_{i}\right)}
$$

and the edge averages by similar expressions. We note that these expressions depend exclusively on the trace of $u$ on the interface $\Gamma$. In these formulas, we could equally well integrate over $\Omega_{j}$ or over $\Omega_{i} \cup \Omega_{j}$. It is easy to see that this new interpolant also reproduces constants as well as the face and edge terms separately. The energy of the face term with the coefficient, given in (10), can be bounded by $\left\|\nabla \mathcal{H}\left(\theta_{\mathcal{F}^{i j}} u\right)\right\|_{L^{2}\left(\Omega_{i}\right)}$. This will result in a bound with two logarithmic factors if we can prove a three dimensional counterpart of Lemma 7.

The analysis of this domain decomposition method is carried out in the framework of the abstract Schwarz theory as in [Toselli and Widlund, 2005, Chapter 2]. If exact solvers are used for the coarse and local problems, each defined on an extended subdomain $\Omega_{i}^{\prime}$, we primarily need a bound on the energy of the coarse interpolant that we have already discussed. There are essentially no new technical difficulties in obtaining bounds for the local terms in the decomposition of an arbitrary finite element function as in [Toselli and Widlund, 2005, Assumption 2.2].

The coarse space can be enriched so that all rigid body modes are exactly reproduced using formulas similar to that of (10). A result for compressible elasticity can then be obtained for two dimensions and John subdomains. The extension to three dimensions will again essentially require the extension of Lemma 7 to edges and faces in three dimensions.

We have established the following result in Dohrmann et al. [2008]. It holds for scalar elliptic problems as well as problems in compressible elasticity.

Theorem 2. Let $\Omega \subset \mathbb{R}^{2}$ be partitioned into nonoverlapping subdomains $\Omega_{i}$, which are John domains, each with a shape regular triangulation. The condition number of our domain decomposition method then satisfies

$$
\kappa\left(P_{a d}\right) \leq C \max _{i}\left(1+H_{i} / \delta_{i}\right)\left(1+\log \left(H_{i} / h_{i}\right)\right)^{2},
$$

where $C$ is a constant which only depends on the John and Poincaré parameters of the subdomains, the number of colors required for the overlapping subdomains, and the shape regularity of the elements. The bound is also independent on variations in the coefficients across the interface $\Gamma$. 


\section{Almost incompressible elasticity}

We also use the same overlapping Schwarz algorithm for almost incompressible elasticity and this is the subject of a paper recently completed. The main emphasis is on regular subdomains, but the result also holds for subdomains that are just John domains and for two dimensions, see [Dohrmann and Widlund, 2008, Section 6]. As previously pointed out, we have only considered mixed finite element methods, with pressure spaces of discontinuous finite element functions. Our main result is:

Theorem 3 (Condition number estimate). The condition number of our domain decomposition method satisfies

$$
\kappa\left(P_{a d}\right) \leq C(1+(H / \delta))^{3}(1+\log (H / h))^{2},
$$

where $C$ is a constant, independent of the number of subdomains and their diameters and the mesh size and which only depends on the number of colors required for the overlapping subdomains and the shape regularity of the elements and the subdomains. The bound is also independent on variations in the coefficients across the interface $\Gamma$.

We note that an early application of overlapping Schwarz methods to mixed formulations of linear elasticity and Stokes problems is given in Klawonn and Pavarino [1998]. In that work, the coarse spaces were based on the same mixed finite element methods on coarse meshes and both continuous and discontinuous pressure spaces were considered. An analysis of these methods was not provided, but their performance was shown to be quite competitive with block diagonal and block triangular preconditioners, see Klawonn and Pavarino [2000].

The new algorithm uses a coarse space similar to that of (10). Just as for the compressible elasticity case, it is enriched so as to contain all the rigid body modes. In our work, there are a number of new challenges, in particular, results on subdomains with bad aspect ratios are required. In addition, when applying the abstract Schwarz theory, great care has to be taken when constructing the coarse component of the partitioning of the displacement fields and new ideas are also required when partitioning the remaining part of an arbitrary finite element function into local components. For details, see Dohrmann and Widlund [2008].

\section{References}

Gabriel Acosta, Ricardo G. Durán, and María A. Muschietti. Solutions of the divergence operator on John domains. Adv. Math., 206(2):373-401, 2006.

Gennady P. Astrakhatsev. Method of fictitious domains for a second-order elliptic equation with natural boundary conditions. U.S.S.R. Computational Math. and Math. Phys., 18:114-121, 1978.

Bogdan Bojarski. Remarks on Sobolev imbedding inequalities. In Complex analysis, Joensuu 1987, volume 1351 of Lecture Notes in Math., pages 52-68. Springer, Berlin, 1988.

James H. Bramble. A proof of the inf-sup condition for the Stokes equations on Lipschitz domains. Math. Models Methods Appl. Sci., 13(3):361-371, 2003. ISSN 0218-2025. 
James H. Bramble, Joseph. E. Pasciak, and Alfred H. Schatz. The construction of preconditioners for elliptic problems by substructuring. I. Math. Comp., 47(175): 103-134, 1986.

Susanne C. Brenner and Ridgway Scott. The Mathematical Theory of Finite Element Methods. Springer-Verlag, Berlin, Heidelberg, New York, 2002. Second edition.

Stephen Buckley and Pekka Koskela. Sobolev-Poincaré implies John. Math. Res. Lett., 2(5):577-593, 1995.

Clark R. Dohrmann, Axel Klawonn, and Olof B. Widlund. Extending theory for domain decomposition algorithms to irregular subdomains. In Ulrich Langer, Marco Discacciati, David Keyes, Olof Widlund, and Walter Zulehner, editors, Proceedings of the 17th International Conference on Domain Decomposition Methods in Science and Engineering, held in Strobl, Austria, July 3-7, 2006, number 60 in Springer-Verlag, Lecture Notes in Computational Science and Engineering, pages 255-261, 2007a.

Clark R. Dohrmann, Axel Klawonn, and Olof B. Widlund. A family of energy minimizing coarse spaces for overlapping Schwarz preconditioners. In Ulrich Langer, Marco Discacciati, David Keyes, Olof Widlund, and Walter Zulehner, editors, Proceedings of the 17th International Conference on Domain Decomposition Methods in Science and Engineering, held in Strobl, Austria, July 3-7, 2006, number 60 in Springer-Verlag, Lecture Notes in Computational Science and Engineering, pages 247-254, 2007b.

Clark R. Dohrmann, Axel Klawonn, and Olof B. Widlund. Domain decomposition for less regular subdomains: Overlapping Schwarz in two dimensions. SIAM J. Numer. Anal., 46(4):2153-2168, 2008.

Clark R. Dohrmann and Olof B. Widlund. An overlapping Schwarz algorithm for almost incompressible elasticity. Technical Report TR2008-912, Department of Computer Science, Courant Institute of Mathematical Sciences, New York University, May 2008. URL: http://cs.nyu.edu/csweb/Research/TechReports/TR2008-912/TR2008-912.pdf.

Maksymilan Dryja and Olof B. Widlund. Some domain decomposition algorithms for elliptic problems. In Linda Hayes and David Kincaid, editors, Iterative Methods for Large Linear Systems, pages 273-291. Academic Press, 1989.

Ricardo G. Durán and María A. Muschietti. The Korn inequality for Jones domains. Electron. J. Differential Equations, 2004(127):1-10, 2004.

Herbert Federer and Wendell H. Fleming. Normal and integral currents. Ann. of Math. (2), 72:458-520, 1960.

Piotr Hajłasz. Sobolev inequalities, truncation method, and John domains. In Papers on analysis, volume 83 of Rep. Univ. Jyväskylä Dep. Math. Stat., pages 109-126. Univ. Jyväskylä, Jyväskylä, 2001.

Peter W. Jones. Quasiconformal mappings and extendability of functions in Sobolev space. Acta Math., 147(1-2):71-88, 1981.

George Karypis and Vipin Kumar. METIS Version 4.0. University of Minnesota, Department of Computer Science, Minneapolis, MN, 1998.

Axel Klawonn and Luca F. Pavarino. Overlapping Schwarz methods for mixed linear elasticity and Stokes problems. Comput. Methods Appl. Mech. Engrg., 165: 233-245, 1998.

Axel Klawonn and Luca F. Pavarino. A comparison of overlapping Schwarz methods and block preconditioners for saddle point problems. Numer. Lin. Alg. Appl., 7: $1-25,2000$. 
Axel Klawonn and Oliver Rheinbach. A parallel implementation of Dual-Primal FETI methods for three dimensional linear elasticity using a transformation of basis. SIAM J. Sci. Comput., 28(5):1886-1906, 2006.

Axel Klawonn and Oliver Rheinbach. Robust FETI-DP methods for heterogeneous three dimensional elasticity problems. Comput. Methods Appl. Mech. Engrg., 196 (8):1400-1414, 2007.

Axel Klawonn, Oliver Rheinbach, and Olof B. Widlund. An analysis of a FETI-DP algorithm on irregular subdomains in the plane. Technical Report TR2007-889, Department of Computer Science, Courant Institute of Mathematical Sciences, New York University, April 2007. URL: http://cs.nyu.edu/csweb/Research/TechReports/TR2007-889/TR2007-889.pdf. To appear in SIAM J. Numer. Anal.

Axel Klawonn and Olof B. Widlund. Dual-Primal FETI Methods for Linear Elasticity. Comm. Pure Appl. Math., 59:1523-1572, 2006.

Axel Klawonn, Olof B. Widlund, and Maksymilian Dryja. Dual-primal FETI methods for three-dimensional elliptic problems with heterogeneous coefficients. SIAM J. Numer. Anal., 40(1):159-179, April 2002.

Jing Li and Olof B. Widlund. FETI-DP, BDDC, and Block Cholesky Methods. Internat. J. Numer. Methods Engrg., 66(2):250-271, 2006.

Jan Mandel, Clark R. Dohrmann, and Radek Tezaur. An algebraic theory for primal and dual substructuring methods by constraints. Appl. Numer. Math., 54:167193, 2005.

Vladimir G. Maz'ja. Classes of domains and imbedding theorems for function spaces. Soviet Math. Dokl., 1:882-885, 1960.

Jindřich Nečas. Les méthodes directes en théorie des équations elliptiques. Academia, Prague, 1967.

L. Ridgway Scott and Shangyou Zhang. Finite element interpolation of nonsmooth functions satisfying boundary conditions. Math. Comp., 54(190):483-493, 1990.

Andrea Toselli and Olof Widlund. Domain Decomposition Methods - Algorithms and Theory, volume 34 of Springer Series in Computational Mathematics. SpringerVerlag, Berlin Heidelberg New York, 2005.

Olof B. Widlund. An extension theorem for finite element spaces with three applications. In Wolfgang Hackbusch and Kristian Witsch, editors, Numerical Techniques in Continuum Mechanics, pages 110-122, Braunschweig/Wiesbaden, 1987. Notes on Numerical Fluid Mechanics, v. 16, Friedr. Vieweg und Sohn. Proceedings of the Second GAMM-Seminar, Kiel, January, 1986. 